\title{
Piezomagnetic Quantum Dots
}

\author{
Ramin M. Abolfath ${ }^{1}$, Andre Petukhov ${ }^{2}$, and Igor Žutić ${ }^{1}$ \\ 1 Department of Physics, State University of New York at Buffalo, Buffalo, New York 14260, USA \\ 2 Department of Physics, South Dakota School of Mines and Technology, Rapid City, SD 57701-3995
}

\begin{abstract}
We study the influence of deformations on magnetic ordering in quantum dots doped with magnetic impurities. The reduction of symmetry and the associated deformation from circular to elliptical quantum confinement lead to the formation of piezomagnetic quantum dots. The strength of elliptical deformation can be controlled by the gate voltage to change the magnitude of magnetization, at a fixed number of carriers and in the absence of applied magnetic field. We reveal a reentrant magnetic ordering with the increase of elliptical deformation and suggest that the piezomagnetic quantum dots can be used as nanoscale magnetic switches.

PACS numbers: 75.75.+a,75.50.Pp,85.75.-d
\end{abstract}

Quantum dots (QDs) can be viewed as artificial atoms which allow for a versatile control of the number of carriers, their spin, and the effects of quantum confinement [1, 2, 3]. The motivation to magnetically dope semiconductor QDs comes from the possibility for an enhanced control of the magnetic ordering as compared to their bulk-like counterparts [4, 5, 6, 7] as well as to directly study the influence of quantum confinement and strong Coulomb interactions on magnetism. There is an encouraging experimental progress in Mn-doped II-VI and III-V QDs, from the controlled inclusion of a single Mn impurity to the onset of magnetization at temperatures substantially higher than in similar bulk materials $8,19,10,11,12,13]$. However, most of the theoretical studies have been limited to a small number of carriers $(N)$ and Mn-impurities $\left(N_{m}\right)$ and the effects of the symmetry of quantum confinement and electron-electron (ee) Coulomb interaction on the magnetic phase diagram, remain to be understood [14, 15, 16, 17, 18]. In particular, it would be useful to know what type of external influences can effectively produce magnetic ordering of carrier spins and magnetic impurities and how such ordering would modify the electrical, optical, and transport properties of QDs.

In the absence of magnetic doping, circular QDs with rotational symmetry of the lateral confining potential have degenerate energy levels and show a pronounced shell structure, similar to atoms and nuclei. The interplay of the Pauli principle and the reduction of e-e Coulomb exchange energy near degenerate levels in the single-particle spectrum, according to Hund's first rule, favors the parallel spin alignment of electrons in open shells [1, 2]. Breaking the circular symmetry by deforming the lateral confinement removes the single-particle level degeneracies and leads to transitions in the spin of electrons, referred to as piezomagnetism [1, 19]. In vertical QDs, elliptical deformation can be made by micronsized device mesas incorporating dots, where the lateral electrostatic confinement originates from side-wall depletion. Both the symmetry of the confinement and the effective QD size can be controlled by the action of a
Schottky gate wrapped around the mesa in the vicinity of the QD [19, 20, 21]. In lateral QDs, the confinement potential is formed electrostatically by several gate voltages, altering the shape and the deformation of the confining potential while keeping $N$ constant [22, 23].

We focus here on the effects of elliptical deformations on magnetic ordering in (II,Mn)VI QDs. In particular, we are interested to explore the onset of a spin-polarized state with finite Mn-magnetization, in the absence of applied magnetic field, to which we refer as "ferromagnetic" (FM) state [14, 15, 16]. Since Mn is isoelectronic with group-II elements, the number of carriers can be changed by chemical doping or by electrostatic gates. We represent magnetic QD in zero magnetic field by the Hamiltonian $H=H_{e}+H_{e x}+H_{m}$, describing contributions of interacting electrons, electron-Mn (e-Mn) exchange, and direct Mn-Mn antiferromagnetic (AFM) coupling, respectively. The interacting electron contribution is given by

$$
H_{e}=\sum_{i=1}^{N}\left[-\frac{1}{2 m^{*}} \nabla_{i}^{2}+U_{Q D}\left(\mathbf{r}_{i}\right)\right]+\frac{e^{2}}{2 \epsilon} \sum_{i \neq j} \frac{1}{\left|\mathbf{r}_{i}-\mathbf{r}_{j}\right|},
$$

where we set $\hbar \equiv 1, m^{*}$ is the electron effective mass, $\mathbf{r} \equiv(\vec{\rho}, z)$, and $U_{Q D}(\mathbf{r})=V_{Q D}(\vec{\rho})+V_{Q D}^{1}(z)$ is the three-dimensional (3D) QD confining potential, where $V_{Q D}^{1}(z)=m^{*} \Omega^{2} z^{2} / 2$ is the $1 \mathrm{D}$ parabolic potential with the characteristic subband energy $\Omega$, and

$$
V_{Q D}(\vec{\rho}) \equiv V_{Q D}(x, y)=(1 / 2) m^{*} \omega_{0}^{2}\left(x^{2} \delta+y^{2} \delta^{-1}\right),
$$

is the 2D anisotropic parabolic potential [1] which describes the lateral confinement with frequencies $\omega_{x}=$ $\omega_{0} \sqrt{\delta}, \omega_{y}=\omega_{0} / \sqrt{\delta}$, where $\delta \equiv \omega_{x} / \omega_{y} \neq 1$ is the strength of elliptical deformation. We impose $\omega_{0}^{2}=\omega_{x} \omega_{y}$ which conserves the area of QD with deformation. The last term in Eq. (11) is the repulsive e-e Coulomb interaction screened by the dielectric constant $\epsilon$, and $-e$ is electron charge. The e-Mn exchange contribution is $H_{e x}=-J_{s d} \sum_{i, I} \vec{s}_{i} \cdot \vec{M}_{I} \delta\left(\mathbf{r}_{i}-\mathbf{R}_{I}\right)$, where $J_{s d}$ is the exchange coupling between electron spin $\vec{s}_{i}$ at $\mathbf{r}_{i}$ 
and impurity spin $\vec{M}_{I}$ at $\mathbf{R}_{I}$. The Mn Hamiltonian is $H_{m}=\sum_{I, I^{\prime}} J_{I, I^{\prime}}^{A F} \vec{M}_{I} \cdot \vec{M}_{I^{\prime}}$, where $J_{I, I^{\prime}}^{A F}$ is the direct Mn-Mn AFM coupling. The $z$-component of $\vec{M}_{I}$ is $M_{z}=-M,-M+1, \ldots, M$, where we choose $\hat{z}$ as the quantization axis and $M=5 / 2$ for $\mathrm{Mn}$. We use a real space finite-temperature local spin density approximation (LSDA) and the mean filed approximation for Mn spins to avoid computational complexity [17, 24] of exact diagonalization, limited to only a very small number of interacting electrons and magnetic impurities 16 . An effective Hamiltonian describing electrons can be obtained by replacing the $\mathrm{Mn}$ spins, that are randomly distributed, with a classical continuous field $H_{e}^{\mathrm{eff}}=H_{e}-$ $\sum_{i} J_{s d} n_{m} \frac{\sigma_{i}}{2}\left\langle M_{z}\left(\mathbf{r}_{i}\right)\right\rangle$, where $n_{m}$ is the averaged density of Mn, and $\sigma= \pm 1$ for spin up ( $\uparrow)$, and down $(\downarrow)$. Within the mean field approach [17], the magnetization density can be expressed as $\left\langle M_{z}\left(\mathbf{r}_{i}\right)\right\rangle=M B_{M}\left(M b\left(\mathbf{r}_{i}\right) / k_{B} T\right)$ where $B_{M}(x)$ is the Brillouin function [25], $k_{B}$ is the Boltzmann constant, $T$ the absolute temperature, while $b\left(\mathbf{r}_{i}\right)=-J_{\text {eff }}^{A F}\left\langle M_{z}\left(\mathbf{r}_{i}\right)\right\rangle+J_{s d}\left[n_{\uparrow}\left(\mathbf{r}_{i}\right)-n_{\downarrow}\left(\mathbf{r}_{i}\right)\right] / 2$ is the effective field seen by the Mn. The first term in $b\left(\mathbf{r}_{i}\right)$ describes the mean field of the direct Mn-Mn AFM coupling [14] and $n_{\sigma}\left(\mathbf{r}_{i}\right)$ is the spin-resolved electron density.

Following the decomposition of 3D confining potential, we expand the QD wave functions in terms of its planar $\psi_{i \sigma}(\vec{\rho})$ and subband wave function $\xi(z)$ (in typical diskshaped QDs only the first subband is filled). We project $H_{e}^{\text {eff }}$ into a $2 \mathrm{D}$ Hamiltonian by integrating out $\xi(z)$. In LSDA we express the Kohn-Sham (KS) Hamiltonian as

$$
H_{K S}=-\frac{1}{2 m^{*}} \nabla_{\rho}^{2}+V_{Q D}+V_{H}+V_{X C}^{\sigma}-\frac{\sigma}{2} h_{s d},
$$

where the $V_{H}$ is the Hartree potential, $V_{X C}^{\sigma}$ is VoskoWilk-Nusair spin dependent exchange-correlation potential [26], and the exchange spin splitting is given by

$$
h_{s d}(\vec{\rho}, T)=J_{e m} \int d z|\xi(z)|^{2} B_{M}\left(\frac{M b(\vec{\rho}, z)}{k_{B} T}\right) .
$$

where $J_{e m}=J_{s d} n_{m} M$ is the e-Mn exchange coupling. We solve self-consistently the Kohn-Sham equations, $H_{K S} \psi_{i \sigma}(\vec{\rho})=\epsilon_{i \sigma} \psi_{i \sigma}(\vec{\rho})$, where $H_{K S}$ is given by Eq. (3) and $\epsilon_{i \sigma}$ are the KS energies. Our numerical results are illustrated for parameters based on $(\mathrm{Cd}, \mathrm{Mn}) \mathrm{Te}$ QDs with $J_{s d}=0.015 \mathrm{eV} \mathrm{nm}^{3}, m^{*}=0.106, \epsilon=10.6$ [16], $a_{B}^{*}=5.29 \mathrm{~nm}$, and $R y^{*}=12.8 \mathrm{meV}$ are the effective Bohr radius and Rydberg energy. We choose a QD with $\omega_{0}=25.6 \mathrm{meV}, n_{m}=0.1 \mathrm{~nm}^{-3}, J_{\text {eff }}^{A F}=0.02 \mathrm{meV}$, and having the perpendicular $(z)$ width $1 \mathrm{~nm}$.

We first examine the effects of elliptical deformation, described with the anisotropic 2D lateral confinement from Eq. (2), on the single-particle levels. In the absence of e-e Coulomb interaction and magnetic impurities $J_{e m}=n_{m}=0$, the corresponding single-particle spectrum $E_{n_{x}, n_{y}}(\delta)=\omega_{0}\left[\left(n_{x}+1 / 2\right) \sqrt{\delta}+\left(n_{y}+1 / 2\right) / \sqrt{\delta}\right]$, where $n_{x(y)}=0, \pm 1, \pm 2, \cdots$, is shown as a function of
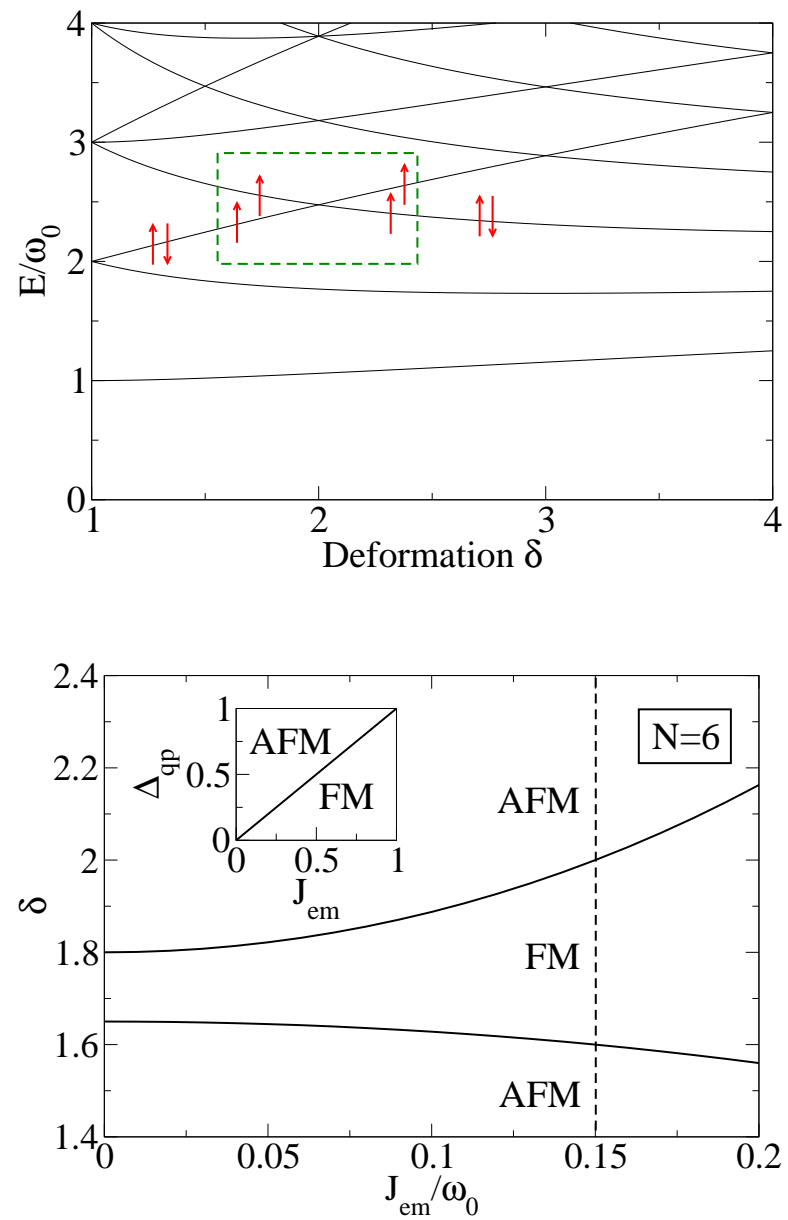

FIG. 1: Effects of elliptical deformation. Top: Single-particle levels of the anisotropic QD as a function of deformation $\delta$. Degeneracies lead to the formation of closed shells with $N=2,6,12,20, \ldots$ in the isotropic (circular) case $(\delta=1)$, and the formation of subshells at $\delta=q / p$ for integer $q, p$. Broken square and the corresponding arrows schematically illustrate how the spin alignment for $N=6$ would be modified near the degeneracy point at $\delta=2, E / \omega_{0}=2.5$ in the presence of e-e Coulomb interaction and magnetic impurities. Bottom: The inset illustrates the magnetic phase diagram as a function of quasi-particle gap $\Delta_{q p}$, and e-Mn exchange coupling $J_{e m}$. The line $\Delta_{q p}=J_{e m}$ separates ferromagnetic (FM) and antiferromagnetic (AFM) ordering. The magnetic phase diagram of QD with $N=6$ interacting electrons at $T=0.5 \mathrm{~K}$ is calculated as a function of $\delta$ and $J_{e m} / \omega_{0}$. The dashed line corresponds to $n_{m}=0.1 \mathrm{~nm}^{-3}$ and $\omega_{0}=25.6 \mathrm{meV}$.

deformation $\delta$ in Fig. 11 top. Isotropic QD $(\delta=1)$ have a pronounced shell structure with $n_{x}+n_{y}+1$-fold degeneracy in each shell, having $N=2,6,12,20, .$. electrons. While, any deviation from the isotropic parabolic confinement breaks the geometrical symmetry and removes these degeneracies, additional accidental degeneracies, can occur in deformed QD at $\delta=p / q$ for integer $p, q$ and lead to the formation of subshells. For example, at $\delta=2$ 


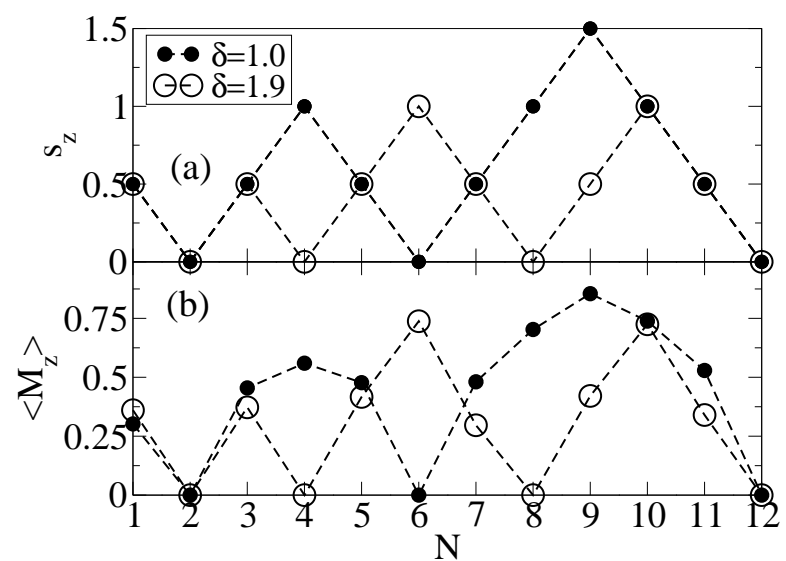

FIG. 2: The $z$-component of the total spin of electrons $s_{z}$ (a) and averaged magnetization per unit area $\left\langle M_{z}\right\rangle$ (b) as a function of number of electrons $N$ at $T=0.5 \mathrm{~K}$ and the Mn-density $n_{m}=0.1 \mathrm{~nm}^{-3}$. Isotropic ( $\delta=1$, filled circles) and elliptical QD $(\delta=1.9$, empty circles) show qualitatively different dependence of $s_{z}$ and $\left\langle M_{z}\right\rangle$ with $N$.

both $p$ - and $d$-, as well as $d$ - and $f$-levels cross, as shown in Fig. 1, top. The $\delta=2$ shell structure is significantly modified from the isotropic case, $N=2,4,8,12, \ldots$, correspond to the closed shells and $N=6,10,15, \ldots$, to the half-filled shells.

For the study of magnetic ordering in (II,Mn)VI QDs, it is important to understand how this single-particle picture is modified in the presence of e-e Coulomb interaction and e-Mn exchange coupling $J_{e m}$. The corresponding sequence of filling electrons and their spin alignment, through carrier-mediated magnetism, affects the magnetic ordering of Mn-impurities and the possibility to externally control Mn-magnetization.

A simple sketch of anticipated modification near $\delta=2$ for the $N=6$ state, shows that the parallel spin alignment of the valence electrons is favored for a wide range of deformation. Additional analysis is addressed in Fig. 1. bottom. The inset shows that the occurrence of FM or AFM state (having finite and vanishing magnetization, respectively), depends on the competition between the two characteristic energy scales: the quasi-particle energy gap $\Delta_{q p}$, and $J_{e m}$ [27]. The single-particle gap should be distinguished from $\Delta_{q p}$ which is the energy difference between highest occupied and lowest unoccupied KS levels and depends on $N$, the total spin of electrons, the strength of e-e Coulomb interaction, and the underlying symmetries of QD confining potential. For $J_{e m}=0$, as shown in Fig. 1 the FM state is stable only within a narrow range of $\delta$ which becomes vanishingly small for noninteracting electrons (at $\delta=2$, Fig. 1 top). The increase in $J_{e m}$ extends the deformation range over which the FM state is possible.

To further examine the effect of elliptical deforma- tion on magnetic ordering we introduce the $z$-component of the total spin of electrons, $s_{z}=\left(N_{\uparrow}-N_{\downarrow}\right) / 2$, and the spatially-averaged Mn-magnetization per unit area $A,\left\langle M_{z}\right\rangle=(1 / A) \int d^{2} \rho\left\langle M_{z}(\vec{\rho})\right\rangle$, obtained from the selfconsistent solution of Kohn-Sham equations. In Fig. 2 we show $s_{z}$ and $\left\langle M_{z}\right\rangle$ as a function of $N$ for both isotropic QD with circular symmetry $(\delta=1)$, and elliptical QD with $\delta=1.9$. For interacting electrons and finite $J_{e m}$ we observe that carrier-mediated magnetism leads to coupled ordering of electron and Mn spins. The oscillations of $s_{z}$ and $\left\langle M_{z}\right\rangle$ reveal that the shell structure is strongly modified with deformation. The vanishing of $s_{z}$ and $\left\langle M_{z}\right\rangle$ correspond to the closed shells $(N=2,6,12, \ldots$, at $\delta=1$ and $N=2,4,8,12, \ldots$, at $\delta=1.9)$ while their local maxima correspond to the half-filled shells $(N=1,4,9, \ldots$, at $\delta=1$ and $N=1,3,6,10, \ldots$, at $\delta=1.9$ ).

Focusing on the $N=6$ state, we see that the minima in $s_{z}$ and $\left\langle M_{z}\right\rangle$ at $\delta=1$ become maxima at the deformation $\delta=1.9$. For an isotropic QD the $p$-levels are degenerate (Fig. 1) and $N=6$ corresponds to the AFM state with $\Delta_{q p}>J_{e m}$ and vanishing $\left\langle M_{z}\right\rangle$. Elliptical deformation breaks the circular symmetry of the lateral confinement and removes the $p$-level degeneracy. At $\delta=1.9, J_{e m}>\Delta_{q p}$ stabilizes the FM state with finite $\left\langle M_{z}\right\rangle$ and $s_{z}=1$ implies that the electron spin polarization is $P=\left(N_{\uparrow}-N_{\downarrow}\right) /\left(N_{\uparrow}+N_{\downarrow}\right)=(4-2) / 6$. The $N=6$ state illustrates an intriguing possibility to turn the Mn-magnetization on and off without changing the number of carriers, needed in the bulk-like magnetic semiconductors. However, $N=6$ is not the unique state, a similar behavior near $\delta=1.5$ is expected also for the $N=12$ state, forming another closed shell in the limit of isotropic confinement. Elliptical deformations of the lateral confinement in Mn-doped QDs thus lead to the change between the AFM and FM state which can be controlled by gate voltage. Such structures would behave as piezomagnetic QDs in which the control of deformation determines the magnetic ordering.

To examine if the piezomagnetic QDs could function as nanoscale magnetization switches, we also consider the evolution of their magnetic ordering with $T$ and $\delta$, shown in Fig. 3 for $N=6$. While $\left\langle M_{z}\right\rangle$ and $P$ monotonically decay with $T$, the inset reveals a nonmonotonic behavior of $\left\langle M_{z}\right\rangle$ and $P$ with $\delta$. The reentrant magnetic ordering with the increase of elliptical deformation corresponds to the AFM-FM-AFM transitions, anticipated from the sketch in Fig. 1 top. Since the change between vanishing and finite $\left\langle M_{z}\right\rangle$ occurs in a narrow range of $\delta$ (near 1.7 or 2.1 , respectively) we expect that a moderate change in the gate voltage would suffice to implement such a magnetization switch [28]. With the increase of $\delta$ we can also anticipate FM-AFM-FM transitions. In the limit of $\delta=1$, a QD with $N=10$ forms the FM state with finite $\left\langle M_{z}\right\rangle$ and $s_{z}=1$ but with an increased deformation and opening up of $\Delta_{q p}$, near $\delta=1.5$, the AFM state with $\left\langle M_{z}\right\rangle=s_{z}=0$ is favored. With the further increase in 


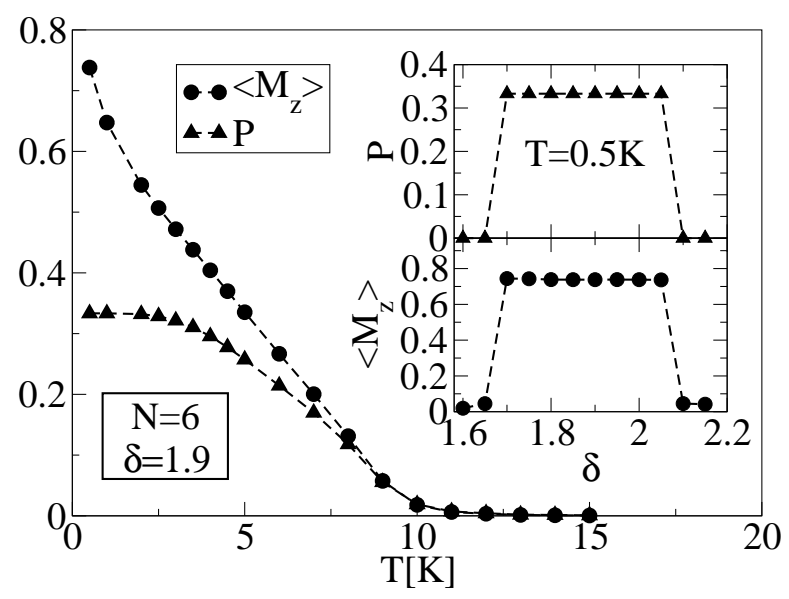

FIG. 3: Temperature evolution of Mn-magnetization per unit area $\left\langle M_{z}\right\rangle$ and electron spin polarization $P$ in an elliptical QD with $\delta=1.9$ and $N=6$. Inset: Evolution of $\left\langle M_{z}\right\rangle$ and $P$ with elliptical deformation at $T=0.5 \mathrm{~K}$ shows a nonmonotonic behavior with sharp rise and fall (near $\delta=1.7$ and $\delta=1.9$, respectively) corresponding to the switching between the AFM and FM state which could be controlled by gate voltage.

$\delta$, we find switching back to the FM state with $s_{z}=1$, near $\delta=2$, also expected from a single-particle picture (Fig. 1, top) and the crossing of $d$ - and $f$-levels at $\delta=2$.

Another potential application of piezomagnetic QDs could be their voltage-controlled $P$ to enable an efficient and tunable spin injection in semiconductor nanostructures [7]. While for the $N=6$ state the total electron spin polarization is limited to $P=(4-2) / 6=1 / 3$, the transfer of only valence electrons could lead to the injection of completely spin-polarized carriers, in the absence of applied magnetic field. Analogously, such QDs could also be used as voltage-controlled spin filters [29].

In conclusion, we have investigated the effects of elliptical deformation, electron-electron Coulomb interaction, and the number of carriers on the magnetic ordering in Mn-doped QDs. We reveal additional possibilities to control magnetism in semiconductor nanostructures which would not be feasible in their bulk counterparts. The two previous experimental results: (1) the feasibility of large elliptical deformations without the Mn-doping 19] and (2) controlled Mn-doping in QDs 8, 9, 10, 11, 12, 13], would need to be combined in order to realize the predicted behavior of piezomagnetic QDs. While our analysis has focused on the electrons and II-VI materials, much larger hole-Mn exchange coupling [25, 30] in either II-VI or III-V QDs could extend the suggested control and manipulation of magnetic ordering to significantly higher temperatures and enable utilization of piezomagnetic QDs for high-density magnetic memory.
This work is supported by the US ONR, NSF-ECCS CAREER, the CCR at SUNY Buffalo, and the Center for Nanophase Materials Sciences, sponsored at ORNL by the Division of Scientific User Facilities US DOE. We thank P. Hawrylak and D. G. Austing for stimulating discussions.

[1] S. M. Reimann and M. Manninen, Rev. Mod. Phys. 74, 1283 (2002), and the references therein.

[2] L. Jacak et al., Quantum Dots (Springer, Berlin, 1998); E. Borovitskaya and M. S. Shur (Eds.), Quantum Dots (World Scientific, New Jersey, 2002).

[3] S. Tarucha et al., in Concepts in Spin Electronics, edited by S. Maekawa (Oxford University Press, Oxford, 2006).

[4] H. Ohno et al., Nature (London) 408, 944 (2000); D. Chiba et al., Science 301, 943 (2003).

[5] S. Koshihara et al., Phys. Rev. Lett. 78, 4617 (1997); A. Oiwa et al., Phys. Rev. Lett. 88, 137202 (2002); H. Boukari et al., Phys. Rev. Lett. 88, 207204 (2002).

[6] T. Jungwirth et al., Rev. Mod. Phys. 78, 1311 (2006); T. Dietl et al., Phys. Rev. B 63, 195205 (2001); S. C. Erwin and I. Žutić, Nature Mater. 3, 410 (2004).

[7] I. Žutić et al., Rev. Mod. Phys. 76, 323 (2004).

[8] S. Mackowski et al., Appl. Phys. Lett. 84, 3337 (2004).

[9] L. Besombes et al., Phys. Rev. Lett. 93, 207403 (2004); Phys. Rev. B 71, 161307 (2005).

[10] Y. Léger et al., Phys. Rev. Lett. 97, 107401 (2006); Phys. Rev. Lett. 95, 047403 (2005).

[11] C. Gould et al., Phys. Rev. Lett. 97, 017202 (2006).

[12] S. Chakrabarti et al.,, Nano Lett. 5, 209 (2005).

[13] P. I. Archer et al.,, Nano Lett. 7, 1037 (2007).

[14] J. Fernández-Rossier and L. Brey, Phys. Rev. Lett. 93, 117201 (2004). J. Fernández-Rossier, Phys. Rev. B 73, $045301(2006)$.

[15] A. O. Govorov, Phys. Rev. B 72, 075358 (2005); Phys. Rev. B 72, 075359 (2005).

[16] F. Qu and P. Hawrylak, Phys. Rev. Lett. 95, 217206 (2005); Phys. Rev. Lett. 96, 157201 (2006).

[17] R. M. Abolfath et al., Phys. Rev. Lett. 98, 207203 (2007).

[18] W. Zhang et al., cond-mat/0608284.

[19] D. G. Austing et al., Phys. Rev. B 60, 11514 (1999).

[20] P. Matagne et al., Phys. Rev. B 65, 085325 (2002).

[21] S. Tarucha et al., Phys. Rev. Lett. 77, 3613 (1996).

[22] J. Kyriakidis et al., Phys. Rev. B 66, 035320 (2002).

[23] D. M. Zumbühl et al., Phys. Rev. Lett. 93, 256801 (2004).

[24] The size of the Hamiltonian matrix to be diagonalized for just $10 \mathrm{Mn}(\operatorname{spin}-5 / 2)$ is $6^{10} \approx 6 \times 10^{7}$.

[25] J. K. Furdyna, J. Appl. Phys. 64, R29 (1988).

[26] C. Dharma-wardana, and F. Perrot in Density Functional Theory, Edited by E. K. U. Gross, and R.M. Dreizler (Plenum Press, New York, 1995).

[27] The smallness of Mn-Mn coupling in (Cd,Mn)Te QDs simplifies this analysis.

[28] D. G. Austing, private communication.

[29] A. L. Efros et al., Phys. Rev. Lett. 87, 206601 (2001).

[30] A. K. Bhattacharjee and C. Benoit à la Guillaume, Phys. Rev. B 55, 10613 (1997). 